\title{
Parasites digestifs des poules villageoises vendues à Ngaoundéré (Cameroun)
}

\author{
Souley Bagari Iya ${ }^{1 *}$ Abdoulmoumini Mamoudou ${ }^{2}$ Bello \\ Maliki Ibrahim ${ }^{1}$ Abass Chahdini Gbambie ${ }^{3}$ Samuel Abah ${ }^{4}$ \\ Farikou Oumarou ${ }^{4}$ Bello Mohaman ${ }^{4}$ Charles Awé ${ }^{5}$ Linda \\ Stella Mbassi ${ }^{1}$ Michel Lontsi-Demano ${ }^{3}$
}

\section{Mots-clés}

Gallus gallus, volaille, parasitisme, helminthe, Coccidia, Cameroun

(C) S. Bagari Iya et al., 2021

\section{(c) (i)}

https://creativecommons.org/licenses/by/4.0/

Submitted: 29 March 2020

Accepted: 6 September 2021

Published: 20 December 2021

DOI: $10.19182 /$ remvt.36800

\section{Résumé}

Une étude a été menée sur le parasitisme digestif des poules villageoises collectées dans deux marchés de la ville de Ngaoundéré (Cameroun) entre mai et août 2018. Neuf genres (Eimeria sp., Raillietina sp., Capillaria sp., Choanotaenia sp., Gongylonema sp., Amoebotaenia sp., Hymenolepis sp., Tetrameres sp. et Echinostoma sp.) et trois espèces (Ascaridia galli, Heterakis gallinarum et Trichostrongylus tenuis) ont été identifiés. Sur les 429 échantillons examinés, 362 étaient porteurs d'un ou de plusieurs parasites, soit un taux d'infestation de 84,4\%. Ce taux était de 86,3\% chez les 240 oiseaux examinés par coproscopie, et de 82,0\% chez les 189 examinés par autopsie. A la coproscopie, les oiseaux adultes étaient plus infestés que les jeunes par Ascaridia galli, Raillietina sp. et Eimeria sp. De même, à l'autopsie une plus forte prévalence de Capillaria sp. et Raillietina sp. a été observée chez les adultes. La plupart des helminthes parasitaient l'intestin grêle. Aucune lésion coccidienne n'a été observée le long du tractus intestinal. Diverses associations de parasites ont été observées avec celle prédominante de Eimeria sp. L'intensité moyenne d'infestation par Eimeria sp., estimée par la méthode de détermination du nombre d'œufs par gramme de fèces de McMaster, a été la plus élevée chez les volailles adultes.

- Comment citer cet article : Bagari Iya S., Mamoudou A., Maliki Ibrahim B., Chahdini Gbambie A., Abah S., Oumarou F., Mohaman B., Awé C., Mbassi L.S., Lontsi-Demano M., 2021. Digestive parasites of local hens sold in Ngaoundere (Cameroon). Rev. Elev. Med. Vet. Pays Trop., 74 (4): 231-235, doi: 10.19182/remvt.36800

\section{INTRODUCTION}

Avec un taux d'accroissement démographique de $3 \%$ par an, l'élevage bovin ne peut à lui seul subvenir aux besoins en alimentation carnée de la populations camerounaise, qui s'élèvent à 46 kilogrammes de viande par habitant et par an (Ngandeu et Ngatchou, 2006). Jusqu'à récemment, les politiques étaient pourtant focalisées

\footnotetext{
1. Institut de recherche agricole pour le développement, Centre de recherche agricole de Wakwa, BP 65, Ngaoundéré, Cameroun.

2. Département de parasitologie et de pathologies parasitaires, Ecole des sciences et de médecine vétérinaire, Université de Ngaoundéré, Ngaoundéré, Cameroun.

3. Unité de recherche de biologie et d'écologie appliquées, Faculté des sciences, Université de Dschang, Dschang, Cameroun.

4. Laboratoire d'entomologie médicale et vétérinaire, Mission spéciale

d'éradication des glossines, Ngaoundéré, Cameroun.

5. Laboratoire national vétérinaire, Garoua, Cameroun.

* Auteur pour la correspondance

Tél. : +237694688847 ; email : iyasouley@yahoo.fr
}

sur le développement du secteur des ruminants pour couvrir ces besoins. Cependant, face aux faibles résultats obtenus, les stratégies de développement ont été réorientées vers la production d'animaux à cycle court, notamment la poule (Ngandeu et Ngatchou, 2006). L'aviculture industrielle est ainsi une activité d'élevage en nette croissance en Afrique subsaharienne, mais l'aviculture traditionnelle reste la plus répandue et joue un rôle-clé dans la quête de l'autosuffisance et de la durabilité de la sécurité alimentaire (Ayssiwede et al., 2011).

La viande de poulet occupe donc une place importante dans l'alimentation des populations africaines en général et camerounaise en particulier. Toutefois, le déficit en produits issus de volailles persiste dans plusieurs pays en développement et particulièrement en Afrique subsaharienne. Cette carence est surtout due aux faibles niveaux de productivité des poules et coqs villageois qui ne bénéficient d'aucun suivi sanitaire (Gueye, 1998). Bien que les maladies parasitaires ne soient pas le plus souvent des causes directes de mortalités chez ces volailles, elles sont néanmoins à l'origine de pertes économiques considérables en raison des baisses de productivité consécutives à l'infestation des animaux 
(Amoussou, 2007). Il est donc important de maîtriser ces affections, ce qui passe nécessairement par la connaissance des agents pathogènes en cause. Or, la dernière étude menée sur les helminthes digestifs des poules et coqs villageois au Cameroun, réalisée dans la ville de Dschang à l'ouest du pays, remonte à 25 ans (Mpoame et Agbede, 1995). A Ngaoundéré, il n'existe aucune étude sur les helminthes digestifs et les coccidies des poules villageoises. L'objectif de nos travaux était ainsi de réaliser un premier inventaire des genres et espèces parasitant les poules villageoises élevées dans cette région du nord du Cameroun.

\section{MATERIEL ET METHODES}

\section{Collecte du matériel animal}

Au total 429 tractus digestifs de poules (ce terme désigne en général dans cet article à la fois les mâles et les femelles) villageoises de races indéterminées ont été collectés de façon aléatoire durant la saison pluvieuse entre mai et août 2018 dans deux marchés de la ville de Ngaoundéré (région de l'Adamaoua, $1100 \mathrm{~m}$ d'altitude, $7^{\circ} 3^{\prime} \mathrm{N}, 13^{\circ} 4^{\prime} \mathrm{E}$ ). Après abattage et dépeçage des volailles, les viscères ont été placés dans des sacs en plastique identifiés, stockés dans un sac isotherme contenant de la carboglace, puis acheminés au laboratoire de parasitologie de la Mission spéciale d'éradication des glossines où ils ont été conservés entre 4 et $8^{\circ} \mathrm{C}$ jusqu'à leur examen (Chollet et al., 1994). Le sexe et l'âge des volailles (trois mois au plus pour les juvéniles et plus de trois mois pour les adultes) ont été déterminés par observation des caractères phénotypiques (FAO, 2013). Les échantillons ont été analysés soit par coproscopie, soit par autopsie helminthologique. Dans un premier temps (mai à mi-août), chaque méthode a été utilisée alternativement un jour sur deux. Pendant les quinze derniers jours de l'étude, seule la coproscopie a été employée.

\section{Recherche des oufs et des oocystes coccidiens}

Parmi les 429 échantillons collectés, 240 ont été analysés par coproscopie. Deux méthodes qualitatives (sédimentation et flottaison) et une méthode quantitative (technique de McMaster réalisée à partir de $2 \mathrm{~g}$ de fèces mélangés à $60 \mathrm{ml}$ d'eau sursaturée de $\mathrm{NaCl}$ ) ont été utilisées pour la recherche et la quantification des œufs et oocystes dans les matières fécales (Chollet et al., 1994) de ces 240 animaux. La quantité d'œufs par gramme de fèces (opg) a été déterminée par la lecture d'une seule lame de McMaster par volaille.

\section{Collecte et identification des helminthes adultes}

Une autopsie a été pratiquée sur 189 des tubes digestifs collectés. Le tractus digestif de chaque volaille a été étalé sur la paillasse et divisé en quatre portions : œsophage-jabot, proventricule-gésier, intestin grêle (duodénum et jéjunum) et caeca. Chaque portion a été incisée longitudinalement et mise dans une boîte de Petri. La paroi a été lavée et rincée à l'eau du robinet, puis le contenu a été décanté à plusieurs reprises jusqu'à obtention finale d'une suspension claire permettant une collecte aisée des parasites à l'aide d'une loupe binoculaire (Kaufmann et Pfister, 1990).

Les helminthes récoltés ont été placés dans des flacons contenant de l'alcool à $70 \%$. Les nématodes ont été éclaircis à la glycérine pure pendant 24 à $48 \mathrm{~h}$. Les cestodes ont été fixés, plongés dans l'acide acétique pendant 5 à $10 \mathrm{~min}$, puis lavés à l'eau et à l'éthanol à $80^{\circ} \mathrm{C}$. Après éclaircissement, les vers ont été rincés à l'éthanol $70^{\circ} \mathrm{C}$ pendant 5 à 10 min (Meyer et Olsen, 1980 ; Euzeby, 1982). L'identification et le comptage des helminthes ont été faits au microscope photonique en suivant les clés disponibles (Yamaguti, 1963 ; Euzeby, 1963 ; 1966 ; Troncy et al., 1981). Pour les cestodes, seuls les scolex ont été comptés car il y avait de nombreux segments séparés dans les boîtes de Petri.

\section{Analyses des données}

Les données collectées ont été analysées avec le logiciel SPSS version 22.0. Le test de chi-2 a permis de comparer les prévalences en fonction du sexe et de l'âge. Les charges parasitaires, quant à elles, ont été comparées par le test d'analyse de variance (Anova) à deux facteurs. La prévalence apparente $(\mathrm{P})$ et l'intervalle de confiance (IC) à $95 \%$ ont été calculés par les formules suivantes (avec $\mathrm{n}$ la taille d'échantillon) :

$\mathrm{P}=\frac{\text { Nombre d'animaux positifs à une méthode de diagnostic }}{\mathrm{n}}$

$$
\mathrm{IC}=\left[\mathrm{P}-1,96 \times \sqrt{\frac{\mathrm{P} \times(1-\mathrm{P})}{\mathrm{n}}} ; \mathrm{P}+1,96 \times \sqrt{\frac{\mathrm{P} \times(1-\mathrm{P})}{\mathrm{n}}}\right]
$$

\section{RESULTATS}

\section{Parasites identifiés}

Sur les 429 échantillons examinés, il y avait $174(40,6 \%)$ femelles et $255(59,4 \%)$ mâles, et 115 (26,8 \%) juvéniles et 314 (73,2 \%) adultes. Par ailleurs, 362 étaient porteurs d'un ou de plusieurs parasites, ce qui équivaut à un taux d'infestation de 84,4\% (IC : 80,9-87,8\%). Le taux était de 86,3\% (IC : 81,9-90,6\%) pour les 240 oiseaux examinés par coproscopie, et de 82,0\% (IC : 76,5-87,5\%) pour les 189 examinés par autopsie. Il n'y a pas eu de différence $(\mathrm{p}>0,05)$ dans les taux d'infestation en fonction de la méthode d'analyse utilisée.

Trois espèces et neuf genres de parasites ont été observés : Ascaridia galli, Heterakis gallinarum et Trichostrongylus tenuis ; Eimeria sp., Raillietina sp., Capillaria sp., Choanotaenia sp., Gongylonema sp., Amoebotaenia sp., Hymenolepis sp., Tetrameres sp. et Echinostoma $\mathrm{sp}$. (tableau I). Certains parasites ont été plus fréquemment observés par autopsie, comme la plupart des cestodes. A l'inverse, les taux d'infection par les nématodes ont été généralement plus élevés lors des coproscopies. Eimeria sp. a été diagnostiqué exclusivement par coproscopie et a été le parasite le plus fréquemment observé.

A la coproscopie, nous n'avons pas observé de différence significative entre les taux d'infestation des deux sexes, ni entre les oiseaux adultes et jeunes, bien que les premiers aient été généralement plus infestés. A l'autopsie, l'infestation des animaux adultes a été en revanche significativement plus importante $(\mathrm{p}<0,05)$ pour Capillaria sp., Raillietina sp. et Choanotaenia sp.

La plupart des helminthes parasitaient l'intestin grêle (tableau II). Deux genres ont cependant été retrouvés dans l'œsophage et le jabot, un dans le gésier, et deux dans les caeca. Heterakis gallinarum a été retrouvé dans l'intestin grêle et les caeca.

\section{Intensités moyennes d'infestation}

Les poules adultes ont eu une intensité moyenne d'infestation par Eimeria sp., estimée par la détermination des opg (tableau III), légèrement plus importante que les jeunes, mais de façon non significative $(\mathrm{p}=0,36)$. Toutefois cette infestation coccidienne, plus importante chez les sujets âgés, s'est présentée sous forme bénigne, sans symptômes apparents et sans qu'aucune lésion coccidienne n'ait été observée le long du tractus intestinal.

\section{Associations parasitaires chez les poules locales}

Différents types d'associations parasitaires ont été observés au cours de l'étude, le nombre d'espèces et de genres de parasites différents par animal pouvant être de deux, trois ou quatre. Les associations de parasites observées ont été très variables avec plus de 30 associations différentes de deux parasites, 25 de trois parasites et 5 de 
Tableau I : Prévalences des parasites digestifs en fonction du sexe et de l'âge des poules (n=429) pour les échantillons examinés par coproscopie (Copro, $\mathrm{n}=240$ ) et par autopsie (Autops, $\mathrm{n}=189$ ) à Ngaoundéré, Cameroun /// Prevalence of digestive parasites according to sex and age of hens ( $n=429$ ) for samples examined by coproscopy (Copro, $n=240$ ) and necropsy (Autops, $n=189$ ) in Ngaoundere, Cameroon

\begin{tabular}{|c|c|c|c|c|c|c|c|c|c|c|}
\hline & \multicolumn{4}{|c|}{ Sexe } & \multicolumn{4}{|c|}{ Age } & \multicolumn{2}{|c|}{ Total } \\
\hline & \multicolumn{2}{|c|}{ Mâle (\%) } & \multicolumn{2}{|c|}{ Femelle (\%) } & \multicolumn{2}{|c|}{ Adulte (\%) } & \multicolumn{2}{|c|}{ Juvénile (\%) } & \multirow[b]{2}{*}{ Copro } & \multirow[b]{2}{*}{ Autops } \\
\hline & Copros & Autops & Copro & Autops & Copro & Autops & Copro & Autops & & \\
\hline \multicolumn{11}{|l|}{ Nématodes } \\
\hline Ascaridia galli & $36(26,5)$ & $11(9,2)$ & $23(21,1)$ & $5(7,1)$ & $44(25,4)$ & $10(7,1)$ & $15(22,4)$ & $6(12,5)$ & $59(24,6)$ & $16(8,5)$ \\
\hline Heterakis gallinarum & $23(16,9)$ & $14(11,8)$ & $21(20,2)$ & $7(10)$ & $36(20,8)$ & $17(12)$ & $8(11,9)$ & $4(8,3)$ & $44(18,3)$ & $21(11,1)$ \\
\hline Capillaria sp. & $15(11)$ & $17(14,3)$ & $16(15,4)$ & $10(14,2)$ & $26(15)$ & $25(17,8)$ & $5(7,4)$ & $2(4,1)$ & $31(12,9)$ & $27(14,3)$ \\
\hline Tetrameres sp. & $7(5,1)$ & $2(1,7)$ & $9(8,7)$ & 0 & $13(7,5)$ & $2(1,4)$ & $3(4,4)$ & 0 & $16(6,7)$ & $2(1,1)$ \\
\hline Trichostrongylus tenuis & $2(1,4)$ & 0 & $1(0,9)$ & 0 & $3(1,7)$ & 0 & $0(0)$ & 0 & $3(1,3)$ & 0 \\
\hline $\begin{array}{l}\text { Gongylonema sp. } \\
\text { Cestodes }\end{array}$ & $13(9,6)$ & $6(5)$ & $5(4,8)$ & $2(2,8)$ & $11(6,4)$ & $6(4,2)$ & $7(10,4)$ & $2(4,2)$ & $18(7,5)$ & $8(4,2)$ \\
\hline Raillietina sp. & $34(25)$ & $67(56,3)$ & $35(33,7)$ & $38(54,3)$ & $52(30,1)$ & $85(60,3)$ & $17(25,4)$ & $20(41,7)$ & $69(28,8)$ & $105(55,6)$ \\
\hline Choanotaenia sp. & $4(2,9)$ & $15(12,6)$ & $9(8,6)$ & $11(15,7)$ & $10(5,8)$ & $24(17)$ & $3(4,4)$ & $2(4,2)$ & $13(5,4)$ & $26(13,8)$ \\
\hline Amoebotaenia sp. & $13(9,5)$ & 0 & $10(9,6)$ & 0 & $17(9,8)$ & 0 & $6(8,9)$ & 0 & $23(9,6)$ & 0 \\
\hline $\begin{array}{l}\text { Hymenolepis sp. } \\
\text { Trématodes }\end{array}$ & $5(3,7)$ & $6(5)$ & $7(6,7)$ & $3(4,2)$ & $10(5,8)$ & $6(4,2)$ & $2(2,9)$ & $3(6,25)$ & $12(5,0)$ & $9(4,8)$ \\
\hline $\begin{array}{l}\text { Echinostoma sp. } \\
\text { Coccidies }\end{array}$ & 0 & $1(0,7)$ & 0 & 0 & 0 & $1(0,7)$ & 0 & 0 & 0 & $1(0,5)$ \\
\hline Eimeria sp. & $79(58,9)$ & 0 & $52(50)$ & 0 & $96(55,4)$ & 0 & $35(52,2)$ & 0 & $131(54,6)$ & 0 \\
\hline
\end{tabular}

Tableau II : Distribution des helminthes digestifs en fonction du site de prédilection chez les poules à Ngaoundéré, Cameroun /// Distribution of digestive helminths according to the site of predilection in hens in Ngaoundéré, Cameroon

\begin{tabular}{|c|c|}
\hline Site de prédilection & Espèce ou genre rencontré \\
\hline CEsophage-jabot & Capillaria sp. et Gongylonema sp. \\
\hline Proventricule-gésier & Tetrameres sp. \\
\hline Intestin grêle & $\begin{array}{l}\text { Ascaridia galli, Raillietina sp., Choanotaenia } \\
\text { sp., Hymenolepis sp., Heterakis gallinarum }\end{array}$ \\
\hline Caeca & Heterakis gallinarum, Echinostoma sp. \\
\hline
\end{tabular}

quatre parasites. Les coccidies ont été impliquées dans la plupart de ces associations, car il s'agissait des parasites les plus fréquents : elles étaient présentes chez $75 \%$ des oiseaux alors que l'helminthe le plus abondant, Raillietina sp., n'a été observé que chez $40 \%$ des animaux.

\section{DISCUSSION}

Les parasites digestifs ont très fréquemment des prévalences élevées chez les volailles villageoises. Cela a été observé dans notre étude $(84,4 \%)$, comme dans celles réalisées dans une autre région du Cameroun (93,5 \% ; Mpaome et Agbede, 1995), au Bénin (86,4\% ;

Tableau III : Intensité moyenne d'infestation des poules par les helminthes digestifs et les coccidies, à Ngaoundéré, Cameroun, estimée par la détermination du nombre d'œufs/oocystes par gramme de fèces (méthode de McMaster) /// Mean infestation intensity of hens with digestive helminths and coccidia in Ngaoundere, Cameroon, estimated by determining the number of eggs/oocysts per gram of feces (McMaster method)

\begin{tabular}{|c|c|c|c|c|}
\hline & \multicolumn{2}{|c|}{ Sexe (moyenne \pm écart-type) } & \multicolumn{2}{|c|}{ Age (moyenne \pm écart-type) } \\
\hline & Mâle & Femelle & Adulte & Juvénile \\
\hline Ascaridia galli & $263 \pm 119$ & $343 \pm 245$ & $315 \pm 209$ & $297 \pm 203$ \\
\hline Heterakis gallinarum & $176 \pm 112$ & $296 \pm 176$ & $258 \pm 161$ & $136 \pm 106$ \\
\hline Capillaria sp. & $266 \pm 121$ & $223 \pm 88$ & $246 \pm 113$ & $238 \pm 48$ \\
\hline Tetrameres sp. & $205 \pm 83$ & $242 \pm 111$ & $200 \pm 84$ & $300 \pm 100$ \\
\hline Trichostrongylus tenuis & $350 \pm 50$ & $200 \pm 0$ & $300 \pm 47$ & 0 \\
\hline Gongylonema sp. & $320 \pm 45$ & $227 \pm 109$ & $259 \pm 128$ & $243 \pm 53$ \\
\hline Raillietina sp. & $311 \pm 180$ & $291 \pm 134$ & $313 \pm 159$ & $265 \pm 154$ \\
\hline Choanotaenia sp. & $261 \pm 171$ & $213 \pm 111$ & $270 \pm 160$ & $167 \pm 104$ \\
\hline Amoebotaenia sp. & $200 \pm 103$ & $212 \pm 98$ & $221 \pm 102$ & $167 \pm 81$ \\
\hline Hymenolepis sp. & $271 \pm 125$ & $200 \pm 71$ & $250 \pm 118$ & $200 \pm 0$ \\
\hline Eimeria sp. & $5625 \pm 4699$ & $5861 \pm 4256$ & $6088 \pm 4470$ & $4847 \pm 4200$ \\
\hline
\end{tabular}


Amoussou, 2007), en Algérie (100\% ; Yousfi, 2012) ou au Niger (100 \% ; Tager-Kagan et al., 1992). L'absence de suivi technique et sanitaire de ces élevages est sans doute une des causes principales de ces observations.

Durant l'étude, deux méthodes d'analyses ont été utilisées, la coproscopie et l'autopsie helminthologique. La combinaison des deux méthodes, même si elle n'a pas été utilisée pour les mêmes échantillons, a permis de faire un diagnostic exhaustif dans la zone d'étude et d'évaluer les lésions potentielles le long du tractus digestif (autopsie). Toutefois, comme les différents échantillons n'ont pas été examinés par les deux méthodes à la fois, il est difficile de faire une comparaison fine entre ces dernières.

Eimeria sp. et Raillietina sp. ont été les parasites les plus fréquemment rencontrés. Ceci peut s'expliquer par le fait que la population étudiée était en majorité adulte. Or, une durée d'élevage prolongée entraîne l'accumulation d'Eimeria sp. chez les oiseaux (Fortineau et Troncy, 1985). Par ailleurs, la coccidiose se présente sous forme bénigne chez les volailles âgées avec des charges faibles et sans symptômes apparents. Ceci est lié à l'immunité protectrice qui se développe avec l'âge et qui existe chez les animaux rustiques (Fortineau et Troncy, 1985 ; Adjovi, 1990). Il est toutefois possible, comme cela a été montré au Bénin, que la coccidiose soit plus sévère chez les poussins (Adjovi, 1990).

Les volailles villageoises, qu'elles soient mâles ou femelles, sont toutes soumises au même risque d'exposition aux infections parasitaires (Kibadi et al., 2019). D'autre part, les animaux de tous les âges (juvéniles et adultes) étaient infestés. Il y avait toutefois des prévalences d'infestation à Capillaria sp., Raillietina sp. et Choanotaenia sp. plus importantes chez les adultes. Ceci pourrait être en rapport avec les habitudes alimentaires des animaux âgés qui consomment plus d'aliments que les jeunes (Amoussou, 2007), ce qui expliquerait aussi l'intensité moyenne d'infestation élevée chez les oiseaux adultes. D'autre part, selon Nalubamba et al. (2015), la volaille adulte est plus insectivore que granivore ce qui peut expliquer une forte prévalence de cestodes et particulièrement de Raillietina $\mathrm{sp}$. car les insectes (et les vers de terre) constituent des hôtes intermédiaires pour plusieurs de ces parasites.

\section{- CONCLUSION}

Cette étude a révélé la présence de trois genres (Capillaria sp., Tetrameres sp. et Gongylonema sp.) et trois espèces (Ascaridia galli, Heterakis gallinarum et Trichostrongylus tenuis) de nématodes, de quatre cestodes (Raillietina sp., Choanotaenia sp., Amoebotaenia sp. et Hymenolepis sp.), d'un trématode (Echinostoma sp.) et d'Eimeria sp. chez les poules vendues au marché de Ngaoundéré, et donc dans les élevages paysans de l'Adamaoua. Des cas d'associations parasitaires ont été observés, impliquant de façon prédominante Eimeria sp. Ce parasitisme interne, de même que le parasitisme externe qu'il faudrait identifier, a-t-il un impact notable sur la productivité des poules villageoises au Cameroun? Il conviendrait de le déterminer pour savoir si une lutte, impliquant des dépenses pour les éleveurs, est nécessaire ou pas.

\section{Remerciements}

Nous tenons à remercier le personnel de la Mission spéciale d'éradication des glossines ainsi que les plumeurs des lieux d'abattage des marchés «Bantaï » et « Petit marché » de la ville de Ngaoundéré pour leur contribution à cette étude.

\section{Déclaration des contributions des auteurs}

SBI, AM, SA, FO et BM ont participé à la conception et à la planification de l'étude ; SBI a recueilli les données de terrain ; SBI et ACG ont effectué les analyses de laboratoire et les analyses statistiques ; SBI, AM et FO ont révisé la première version du manuscrit ; MLD et BMI ont refait les analyses statistiques; CA a participé à la révision de la première correction ; SBI, BMI, CA et LSM ont participé à la deuxième correction du manuscrit.

\section{REFERENCES}

Adjovi A., 1990. L'aviculture traditionnelle Béninoise. In: Proc. CTA Semin. Small holder Rural Poultry Production, Thessaloniki, Greece, 2: 3-11

Amoussou B., 2007. Ectoparasitisme et parasitisme helminthique du poulet local dans le Sud Benin (les Départements de l'Atlantique, du Littoral, de l'Ouémé et du Plateau). Thèse Doct., Ecole inter-Etat des sciences et médecine vétérinaires, Dakar, Sénégal, $85 \mathrm{p}$

Ayssiwede S.B., Dieng A., Houinato M.R.B., Chrysostome C.A.A.M., Issay I., Hornnick J.L., Missohou A.I., 2011. Elevage des poulets traditionnels ou indigènes au Sénégal et en Afrique subsaharienne : état des lieux et contraintes. Ann. Méd. Vét., 157: 103-119

Chollet J.Y., Martrenchar A., Bouchel D., Njoya A., 1994. Epidémiologie des parasitoses digestives des jeunes bovins dans le Nord-Cameroun. Rev. Elev. Med. Vet. Pays Trop., 47 (4) : 365-374, doi : 10.19182/remvt.9073

Euzeby J., 1963. Les maladies vermineuses des animaux domestiques et leurs incidences sur la pathologie humaine. Tome 1, Maladies dues aux némathelminthes. Vigot Frères, Paris, France, $843 p$

Euzeby J., 1966. Les maladies vermineuses des animaux domestiques et leurs incidences sur la pathologie humaine. Tome 2, Maladies dues aux plathelminthes. Vigot Frères, Paris, France, 663 p.

Euzeby J., 1982. Diagnostic expérimental d'helminthoses animales. Livre 2, Diagnostic direct post-mortem, Diagnostic indirect. Informations techniques des services vétérinaires, Paris, France, 364 p.

FAO, 2013. Phenotypic characterization of animal genetic resources. FAO Animal production and heath guidelines, FAO Rome, Italy, 143 p.

Fortineau O., Troncy P.M., 1985. Coccidiose, maladies animales majeures. Les coccidioses du poulet. Rev. Elev. Med. Vet. Pays Trop. Nouvelle Calédonie (2) : 11-18

Gueye E.F., 1998. Village egg and meat production in Africa. World Poult. Sci. J. 54: 73-86, doi: 10.1079/WPS19980007

Kaufmann J., Pfister K., 1990. The seasonal epidemiology of gastrointestinal nematodes in N'Dama cattle in the Gambia. Vet. Parasitol., 37 (1): 45-54, doi: 10.1016/0304-4017(90)90024-6

Kibadi V.M., Malonga B., Mukwela Mutekisel M., 2019. Parasites gastrointestinaux de la poule locale en divagation dans la cellule Ott, quartier Ngulunzamba, ville de Kikwit en République démocratique du Congo. Congosciences, 7 (1) : 182-188

Meyer M.C., Olsen O.W., 1980. Essentials of parasitology, $3^{\text {rd }}$ edn. C. Brown, Dubuque, IA, USA, 303 p.

Mpoame M., Agbede G., 1995. The gastro-intestinal helminth infection of domestic fowl in Dschang, Western Cameroon. Rev. Elev. Med. Vet. Pays Trop., 48 (2), 147-151, doi: 10.19182/remvt.9466

Nalubamba K.S., Bwalya E.C., Mudenda N.B., Munangandu H.M., Munyeme M., Squarre D., 2015. Prevalence and burden of gastrointestinal helminths in wild and domestic guineafowls (Numida meleagris) in the Southern Province of Zambia. Asian Pac. J. Trop. Biomed., 5 (8): 663-670, doi: 10.1016/j. apjtb.2015.04.009

Ngandeu E., Ngatchou A., 2006. Première évaluation du secteur avicole au Cameroun : Structure et importance du secteur avicole commercial et familial pour une meilleure compréhension de l'enjeu de l'influenza aviaire. FAO, Rome, Italy, 48 p.

Tager-Kagan P., Tibayrenc R., Garba, D., 1992. Epidémiologie du parasitisme aviaire en élevage villageois dans la région de Niamey, Niger. Rev. Elev. Med. Vet. Pays Trop., 45 (2) : 139-147, doi : 10.19182/remvt.8940

Troncy P.M., Itard J., Morel P.C., 1981. Précis de parasitologie vétérinaire tropicale. Ministère de la Coopération et du Développement, Paris, France, 717 p.

Yamaguti S., 1963. Systema helminthum, Acanthocephala. Vol. V Interscience. John Wiley, New York, USA, 423 p.

Yousfi F., 2012. Contribution à l'étude des helminthes parasites du tube digestif du poulet local, (Gallus gallus domesticus, Linnaeus, 1758) dans la région d'Oran, Thèse Doct., Algérie, 131 p. 


\section{Summary}

Bagari lya S., Mamoudou A., Maliki Ibrahim B., Chahdini Gbambie A., Abah S., Oumarou F., Mohaman B., Awé C., Mbassi L.S., Lontsi-Demano M. Digestive parasites of local hens sold in Ngaoundere (Cameroon)

A study was conducted on the digestive parasitism of local hens collected in two markets in the city of Ngaoundéré (Cameroon) between May and August 2018. Nine genera (Eimeria sp., Raillietina sp., Capillaria sp., Choanotaenia sp., Gongylonema sp., Amoebotaenia sp., Hymenolepis sp., Tetrameres sp. and Echinostoma sp.) and three species (Ascaridia galli, Heterakis gallinarum and Trichostrongylus tenuis) were identified. Of the 429 samples examined, 362 carried one or more parasites for an infestation rate of $84.4 \%$. This rate was $86.3 \%$ in the 240 birds examined by coproscopy, and $82.0 \%$ in the 189 birds examined by necropsy. At coproscopy, adult birds were more infested than young birds with Ascaridia galli, Raillietina sp. and Eimeria sp. Similarly, at necropsy a higher prevalence of Capillaria sp. and Raillietina sp. was observed in adults. Most helminths were parasitizing the small intestine. No lesions of Coccidia were observed along the intestinal tract. Various associations of parasites were observed, with Eimeria sp. predominating. The average intensity of infestation by Eimeria sp., estimated by McMaster's egg per gram of feces method, was highest in adult birds.

Keywords: Gallus gallus, poultry, parasitism, helminths, Coccidia, Cameroon

\section{Resumen}

Bagari Iya S., Mamoudou A., Maliki Ibrahim B., Chahdini Gbambie A., Abah S., Oumarou F., Mohaman B., Awé C., Mbassi L.S., Lontsi-Demano M. Parásitos digestivos de las gallinas de pueblo vendidas en Ngaoundéré (Camerún)

Se llevó a cabo un estudio sobre el parasitismo digestivo de las gallinas de pueblo recolectadas en dos mercados de la villa de Ngoundéré (Camerún) entre mayo y agosto 2018. Nueve géneros (Eimeria sp., Raillietina sp., Capillaria sp., Choanotaenia sp., Gongylonema sp., Amoebotaenia sp., Hymenolepis sp., Tetrameres sp. y Echinostoma sp.) y tres especies (Ascaridia galli, Heterakis gallinarum y Trichostrongylus tenuis) fueron identificados. Sobre las 429 muestras examinadas, 362 fueron portadoras de uno o más parásitos, o sea una tasa de infestación de $84,4 \%$. Esta tasa fue de $86,3 \%$ en las 240 aves examinadas por examen coprológico y de $82,0 \%$ en las 189 examinadas por autopsia. En el examen coprológico, las aves adultas estaban más infestadas que las jóvenes por Ascaridia galli, Raillietina sp. y Eimeria sp. Igualmente, en la autopsia se observó una prevalencia más fuerte de Capillaria sp. y Raillietina sp. en los adultos. La mayoría de los helmintos parasitaron el intestino delgado. No se observó ninguna lesión por coccidia a lo largo del tracto intestinal. Diversas asociaciones de parásitos fueron observadas, con predominancia de Eimeria sp. La intensidad media de infestación por Eimeria sp., estimada mediante el método de determinación del número de huevos por gramo de heces de McMaster, fue más elevada en los volátiles adultos.

Palabras clave: Gallus gallus, aves de corral, parasitismo, helmintos, Coccidia, Camerún 
